\title{
Validation of the Metastatic Formula: A Method of Cervical Lymph Nodes Assessment in Oral Cancer Patients
}

\author{
Ahmed E Ali ${ }^{1}$, Yousif I Eltohami ${ }^{1 *}$, Mohammed M Elkulaibi ${ }^{2}$ and Ahmed M Suleiman ${ }^{1}$ \\ ${ }^{1}$ Department of Oral and Maxillofacial Surgery, University of Khartoum, Sudan \\ ${ }^{2}$ Department of Oral and Maxillofacial Surgery, Sana'a University, Yemen
}

Submission: November 01, 2018; Published: November 29, 2018

*Corresponding author: Yousif I Eltohami, Assistant professor of Oral and Maxillofacial Surgery, Faculty of dentistry, University of Khartoum, Sudan

Abstract

Background: The Metastasis Score (MS) had been introduced in 2007 as a new method for cervical nodes assessment in oral and maxillofacial cancer patients. The metastasis score (MS) was taken from the CT scan interpretation in the preoperative assessment and was found to be reliable.

Objectives: To validate and evaluate the accuracy of the metastasis score (MS); a new method for cervical lymph nodes ASSESSMENT for metastasis in oral and maxillofacial cancer patients, in comparison to histopathology results.

Materials and Methods: The study was conducted in Khartoum Teaching Dental Hospital, the main oral and maxillofacial referral center, during the period 2011-2013. Clinical investigation, CT scan, the metastasis scores (MS); from the CT scan interpretation, was calculated preoperatively on 25 patients who undergone neck dissection for primary head and neck malignancy.

Results: Seven cases had a score of (0-3) and 18 cases had a score of (6-10). Twelve (48.0\%) cases were positive (+ve) for neck metastasis and $13(53.0 \%)$ cases were negative (-ve) for neck metastasis in the histopathology results. The histopathology results for the cases with metastasis score (MS) (0-3) showed (-ve) results in all the cases with an accuracy of $100 \%$ as there was no (+ve) results. For metastasis score (MS) the group (6-10) the histopathology results were (-ve) in 6 cases with an accuracy of 33.3\% and it was (+ve) in 12 cases with an accuracy of $66.7 \%$. The Sensitivity (true +ve results) and specificity (true -ve results) of this study are $100 \%$ and $53 \%$ respectively.

Conclusion: The metastasis score (MS) predicts cervical metastasis with an accuracy of $100 \%$ for the group (0-3) and with an accuracy of $66.7 \%$ for the group (6-10) as there was an incidence of false positive results; nevertheless, this group mostly present with clinically positive neck where prophylactic neck dissection is indicated.

Keywords: Shade; Compatibility; VITA; Composite

\section{Introduction}

Cancer is a generic term for a large group of diseases that can affect any part of the body. Other terms used for cancer are malignant tumors and neoplasms. It is a leading cause of death worldwide and accounted for 7.6 million deaths (around 13\% of all deaths) in the year 2008. The mortality rate of it is increasing with an estimate of 13.1 million deaths by the year 2030 [1].

The characteristic features in the pathogenesis of cancer is the rapid creation of abnormal cells that grow beyond their usual boundaries and which can then invade adjoining parts of the body and spread to other organs, such spread can be through lymphatic and blood vessels; this process is known as metastasis, which is the major cause of death from cancer [1]

Head and neck cancer (HNC) are the sixth most common cancer globally [2]. HNC includes cancer originating in over 30 specific anatomical sites, most of them occur in the surface layers of the upper aerodigestive tract (UAT), the oral cavity, the upper part of the throat, the respiratory system (pharynx) and the voice box (larynx) [3].

The oropharynx is the third commonest site among males in the developing and industrialized countries, with men affected two to three times as often as women due to alcohol and tobacco use. Alcohol, tobacco use and poor diet taken together are responsible for $90 \%$ of head and neck cancer [4]. In addition, human papilloma virus (HPV) was shown to be associated with the development of a unique papillary type of squamous cell carcinoma (SCC) within the upper aerodigestive tract, HPV-16 accounting for $90 \%-95 \%$ of such cases [5].

In Sudan a recent study showed that oral cancer is the second most occurring cancer among all body cancers [6]. In a previous 
study of 261 cases of cancer, the most common pattern was intraoral squamous cell carcinoma $(73.6 \%)$, with a male to female ratio of approximately 3:2 [7].

Squamous cell carcinoma of the head and neck grows locally and then metastasize to cervical lymph nodes [8]. Cervical lymph nodes metastasis reduces the survival rate by up to $50 \%$ in patients with SCC of the upper aerodigestive tract $[9,10]$. The presence of metastatic cervical lymph nodes is very important in the prognosis and treatment planning of cancer. Cervical lymph nodes should be suggested as metastatic in patient with primary head and neck cancer and treated accordingly. However clinically palpable lymph nodes might not be metastatic and those were not detected clinically might be involved histopathological [10].

Surgical treatment of these oral tumors is excision and neck dissection [8]. The difficulty in predicting the presence of metastatic disease in clinically negative necks lead to wide spread use of elective (prophylactic) neck dissection or radiation [11], increasing the risk of morbidity or even mortality for the patient [10].

Pretreatment evaluation methods, palpation, ultrasonic tomography (USG) and computed tomography scan (CT scan) for the neck staging are significantly different from histopathology results and suggesting that no pretreatment study can accurately assess the requirement to histopathology [10].

Computed Tomography (CT) has been available for over a decade as diagnostic tool to evaluate cervical lymph nodes since 1981 [12]. The Metastatic formula which has been suggested is an index for cervical lymph nodes assessment in oral and maxillofacial cancer patients that allows for a more proper treatment [13].

In this study we aim to assess the validity of this formula among patients of oral and maxillofacial cancer at Khartoum Dental Teaching Hospital. Patients will be scored accordingly, and the score will be correlated with histopathology results.

\section{Materials and Methods}

A prospective descriptive hospital-based study carried out during the period of 2010 to 2013 at Khartoum Teaching Dental Hospital, the main referral center of oral and maxillofacial cancer patients in Sudan. Clinical investigation, CT scan, the Metastasis Scores (MS); from the CT scan interpretation, was calculated preoperatively on 25 patients who undergone neck dissection for primary head and neck malignancy. Exclusion criteria were: Patient with evidence of distant metastasis (M1), surgically inoperable patient who will receive palliative care and patient who are known to be allergic to the CT scan contrast media or those cannot tolerate the contrast media. Data were collected from patients, interpretation of CT scan and histopathology reports. Data were entered in computer using the SPSS software. All statistical analysis was set at $95 \% \mathrm{cl}$, and all test of significance are two sided.

a. Study plan: CT scan interpretation and assessment: All CT scans were taken in axial, coronal, and 3D in fine slices and were evaluated by a single radiologist for the parameters of the metastasis score (MS), named: Number of the lymph nodes per region, Shape of the lymph nodes, Presence or absence of central necrosis, and size of the lymph node.

\section{Metastasis formula}

All the above-mentioned criteria were given a score according to its metastatic possibility. The total of these scores was called metastasis score (MS) = lymph nodes number score + lymph nodes shape score + lymph nodes necrosis score+ lymph nodes size score. Patients fulfilling the inclusion and exclusion criteria were operated in Khartoum Teaching Dental Hospital by a single expert surgeon. Excised tissues were received and sent for histopathology lab in $10 \%$ formaldehyde as follows; container of the excised primary lesion for assessment of free surgical margins and containers labeled according to excised lymph nodes level. All levels of the lymph nodes were processed and examined by single expert histopathologist for the presence or absence of malignancy. Presence of the tumor deposits in any lymph node defines the entire neck as positive for metastasis.

Ethical issues: Ethical approval was obtained from the Ethical Committee at Sudan Medical Council and Research Committee Review Board at the Faculty of Dentistry University of Khartoum and from the General Directorate of Khartoum Teaching Dental Hospital. Patients were asked to participate in the study verbally.

Table 1: Postoperative histopathology results.

\begin{tabular}{|c|c|c|}
\hline Histopathology & Frequency & Percent \\
\hline - ve & 13 & 52 \\
\hline + ve & 12 & 48 \\
\hline Total & 25 & 100 \\
\hline
\end{tabular}

Results: Twenty-five cases of oral and maxillofacial cancer patients were investigated for neck metastasis, 12 (48.0\%) cases were positive (+ve) for neck metastasis and 13 (52.0\%) cases were negative (-ve) for neck metastasis in a histopathology result (Table 1).

Table 2: Metastasis score frequency for each patient individually.

\begin{tabular}{|c|c|c|}
\hline MS & Frequency & Percent \\
\hline 0 & 2 & 8 \\
\hline 1 & 1 & 4 \\
\hline 2 & 4 & 16 \\
\hline 6 & 2 & 8 \\
\hline 7 & 3 & 12 \\
\hline 8 & 9 & 36 \\
\hline 9 & 4 & 16 \\
\hline Total & 25 & 100 \\
\hline
\end{tabular}

The frequency for the metastasis score (MS) was taken from each case individually and from the metastasis score (MS) groups as classified by the previous study (Table $2 \& 3$ ) respectively. No patients were found to have the metastasis score (MS) 4 and 5. 
Table 3: Metastasis score frequency for metastasis score group.

\begin{tabular}{|c|c|c|}
\hline (MS) group & Frequency & Percent \\
\hline $0-3$ & 7 & 28 \\
\hline 4 & - & - \\
\hline 5 & - & - \\
\hline 06-Oct & 18 & 72 \\
\hline Total cases & 25 & 100 \\
\hline
\end{tabular}

The histopathology results for the metastasis score group $(0-3)$ were (-ve) in 7 cases with $100 \%$ accuracy as there was no (+ve) results. For the metastasis score (MS) group (6-10) the histopathology results were (-ve) in 6 cases with $33.3 \%$ accuracy and it was (+ve) in 12 cases with $66.7 \%$ accuracy (Table 4).

The results of this study are statistically significant as $\mathrm{P}$ value $=0.03$. The Sensitivity (true +ve results) and specificity (true - ve results) of this study are $100 \%$ and $53 \%$ respectively.

Table 4: Metastasis score group correlated to histopathology results in crosstabulation.

\begin{tabular}{|c|c|c|c|c|}
\hline & \multirow{2}{*}{ Metastasis score group } & \multicolumn{2}{|c|}{ Histopathology Results } & \multirow{3}{*}{$\begin{array}{c}\text { Total } \\
7 \\
\end{array}$} \\
\hline & & \multirow{2}{*}{$\begin{array}{c}\text { - ve } \\
7 \\
\end{array}$} & \multirow{2}{*}{$\begin{array}{c}+ \text { ve } \\
0\end{array}$} & \\
\hline \multirow{4}{*}{$0-3$} & Count & & & \\
\hline & $\%$ within metastasis score group & $100.00 \%$ & $0.00 \%$ & $100.00 \%$ \\
\hline & $\%$ within histopathology results & $53.80 \%$ & $0.00 \%$ & $28.00 \%$ \\
\hline & $\%$ of Total & $28.00 \%$ & $0.00 \%$ & $28.00 \%$ \\
\hline \multirow{4}{*}{$06-0 c t$} & Count & 6 & 12 & 18 \\
\hline & $\%$ within metastasis score group & $33.30 \%$ & $66.70 \%$ & $100.00 \%$ \\
\hline & \% within histopathology results & $46.20 \%$ & $100.00 \%$ & $72.00 \%$ \\
\hline & $\%$ of Total & $24.00 \%$ & $48.00 \%$ & $72.00 \%$ \\
\hline \multirow{4}{*}{ Total } & Count & 13 & 12 & 25 \\
\hline & $\%$ within metastasis score group & $52.00 \%$ & $48.00 \%$ & $100.00 \%$ \\
\hline & $\%$ within histopathology results & $100.00 \%$ & $100.00 \%$ & $100.00 \%$ \\
\hline & $\%$ of Total & $52.00 \%$ & $48.00 \%$ & $100.00 \%$ \\
\hline
\end{tabular}

\section{Discussion}

The status of the cervical lymph nodes is the single most important prognostic factor in head and neck cancer [14]. Knowing whether metastasis is present in the neck is the corner stone in the treatment of patients. Frequently needless neck dissections are performed increasing cost and morbidity of patients. According to Van Den Brakel et al. up to $25 \%$ of patients with squamous cell carcinoma of the head and neck have exclusively micro metastasis [8]. Evidence of metastases in the neck necessitates comprehensive clearance of regional lymphatic basins. However, even if there is no evidence of lymph nodes metastases, when the risk for positive neck lymph nodes exceeds $15-20 \%$ elective neck dissection is indicated [2].

CT scan has been used in the staging of head and neck tumors; evaluating local extension and neck metastasis. The criteria to consider a neck node positive have been described in several publications [8]. The present study is aimed to assess the validity of the metastasis score (MS); a new method for cervical lymph nodes metastasis, designated in a previous study by Elkulibi \& Suleiman and adopted to assess oral and maxillofacial cancer patients at Khartoum Teaching Dental Hospital. The present study is designed to validate and evaluate the accuracy of the metastasis score (MS) in the assessment of cervical lymph nodes metastasis in oral and maxillofacial cancer patients.

Twenty-five patients of oral and maxillofacial cancer had been investigated for cervical lymph nodes metastasis using the metastasis score (MS) and correlated with histopathology results for the presence or absence of metastasis. The findings revealed that $12(48.0 \%)$ cases were positive (+ve) for neck metastasis and $13(52.0 \%)$ case were negative (-ve) for neck metastasis. Two patients (8.7\%) scored 0, 1(4.0\%) patient scored 1, 14 (17.4\%) patients scored 2, $2(8.7 \%)$ patients scored 6, $3(13.0 \%)$ patients scored 7, 9 (39.1\%) patients scored 8 and 4 (16.0\%) patients scored 9. From these findings most of the patients showed high metastasis score (MS). The authors classified the (MS) into groups according to its possibility for nodal metastasis, first group ranging from (0-3), the second group for $(\mathrm{MS})=4$, the third group is for (MS) $=5$ and the final group is for (MS) (6-10). In the present study we have 7 patients $(28.0 \%)$ in the group (0-3) and $18(72.0 \%)$ patients in the group (6-10).

The resultant scores were correlated with the histopathology results with a cross tabulation test using the (SPSS) software. The results showed that the accuracy of the preoperative assessment of the metastatic status of the cervical lymph nodes in patients presenting with oral and maxillofacial cancer using the metastatic score (MS) remains superior to the previous studies and somewhat compatible with the study to be validated. It showed that the sensitivity of the assessment for neck metastasis has improved marginally. This study is consistent with the previous study in the first group (0-3), showing an accuracy of $100 \%$.

Moreover, for the Metastasis Score (MS) in the group (6-10) the histopathology results were (-ve) in 6 cases with an accuracy of $33.3 \%$ and was (+ve) in 12 cases with an accuracy of $66.7 \%$. The histopathology findings in the surgical neck dissections 
provided convenient results associated with the Metastasis Score (MS) results. Our findings showed that the Metastasis Score (MS) can accurately assesses the cervical lymph nodes for metastasis in the group (0-3) with an accuracy of $100 \%$ and for the group (6-10); with an accuracy of $66.7 \%$ and an incidence of $33.3 \%$ false positive results.

In general, these findings are in close agreement with the previous study, i.e. the study to be validated, although the sample doesn't included Metastasis Score (MS) 4 and 5. Nevertheless, it suggests that CT scan when performed in the manner described and interpreted with special attention to the parameters outlined by the previous study, can provide superior information to formulate the Metastasis Score (MS).

The increase in false(+ve) incidence rate in the present study than in the previous study is uncertain although similar methods were used, such as fine slices of the preoperative CT scan, image enhancement with an intravenous contrast medium and all the CT scans were assessed by a single radiologist using the currently recommended diagnostic criteria. The patients underwent surgery as soon as possible after the preoperative assessment to reduce the risk of new tumor growth influencing the results.

Multiple researches had discussed the accuracy of the diagnostic techniques in assessing the cervical nodes metastasis. Techniques that in use for the assessment of cervical lymph nodes metastasis are, clinical palpation, imaging techniques such as CT, MRI, PET CT scan, SLN biopsy and ultrasound-guided fine needle aspiration cytology and all of them had been used to improve upon the results of clinical palpation alone. These diagnostic techniques showed less than $100 \%$ accuracy for neck metastasis and showed lower sensitivity and Somewhat lower specificity, thus the risk of occult disease in the neck will remain [10,15-19].

Martinez-Gimeno Scoring System (MGSS) which permits a risk evaluation for neck metastases in squamous cell carcinoma of the oral cavity is a histopathology-based scoring system. On their study in 2010 the authors showed a high sensitivity of $100 \%$ and specificity of $83 \%$ in comparison to CT scan and clinical palpation [8]. Using the Metastasis Score (MS) which is a clinical method built on a CT scan as a preoperative tool for cervical lymph nodes assessment is easier than using (MGSS) which is a histopathologybased scoring system of multiple complicated parameters. In addition, necrosis of a lymph node (presence of central hypoattenuation in the lymph nodes on CT scan) is a turning point in the buildup of the Metastasis Score (MS). It had been strongly correlated with histopathology results in the previous study as its presence was the major determinant factor for lymph node metastasis histopathological, with $100 \%$ accuracy [20].

The presence or absence of necrosis on the CT scan is critical, as it will add a score of 4 to the formula which will affect the final Metastasis Score (MS) and increasing the possibility for metastasis. For example, if the other parameters of the formula were 0 , a positive necrosis will push the final Metastasis Score (MS) from the first group (0-3), which showed 100\% accuracy for (-ve) metastasis on the histopathology, into group (MS) $=4$, which showed 33\% accuracy for (+ve) metastasis.

This novel method of evaluating the cervical lymph nodes is easy and reliable and allows for a new way to select patients indicated for neck dissection and sparing those which are not indicated for neck dissection. In those cases, in which the probability of metastasis is low we have two options: either a wait and see policy with a close follow up of the patients; or if the patient prefers or it is not possible to do a close follow up, prophylactic neck dissection is indicated. Following this protocol, we can avoid many needless neck dissections in patients with head and neck cancer.

\section{Limitations of the study}

a. The present study was based on small sample of patients, and therefore, the incidence of false (+ve) results for the group (6$10)$ may looks big when presented as a percentage and compared to the previous one.

b. Poor quality CT scans will alter the scoring system as criterion of necrosis is of paramount importance for the final (MS).

\section{Conclusion}

A significant relationship was found between the metastasis score (MS) and the pathologic status of the neck with $100 \%$ sensitivity and 53\% specificity. From the results of the present study the accuracy of the (MS) for predicting (+ve) histopathology results in the metastasis score (MS) group (0-3) were $100 \%$ and was $66.7 \%$ for the group (6-10) as there were false positive results. These findings agree with the findings of the previous study.

\section{Recommendations}

a. Recording of the metastasis score (MS) for any cancer patient which is easy and reliable as a preoperative assessment tool.

b. Metastasis score (MS) is a postoperative reference, as both clinicians and patients will benefit from it.

\section{References}

1. Center media W (2010) Cancer fact sheet.

2. Gil Z, Fliss DM (2009) Contemporary Management of Head and Neck Cancers. IMAJ 11(5): 296-300.

3. Collins R, Flynn A, Melville A, Richardson R, Eastwood A (2005) Effective health care: management of head and neck cancers. Qual Saf Health Care 14(2): 144-148.

4. Johnson N (2001) Tobacco Use and Oral Cancer: A Global Perspective. J Dent Educ 65(4): 328-339.

5. Trotta BM, Pease CS, Rasamny JJ, Raghavan P, Mukherjee S (2011) Oral Cavity and Oropharyngeal Squamous Cell Cancer: Key Imaging Findings for Staging and Treatment Planning. Radiographics 31(2): 339-354.

6. Nazik O, Albashir AMS (2011) Frequency of Oral Cancer among Cancer in OMF pathology.

7. Osman TA, Satti AA, Boe OE, Yang YH, Ibrahim SO, et al. (2010) Pattern of malignant tumors registered at a referral oral and maxillofacial 
hospital in Sudan during 2006 and 2007. J Cancer Res Ther 6(4): 473474.

8. Martínez-Gimeno C1, Rodríguez-Delgado LE, Perera-Molinero A Trujillo Mdel C, Chivite A, et al. (2011) A new method for the prediction of cervical node metastases in squamous cell carcinoma of the oral cavity: A combination of Martínez-Gimeno Scoring System and clinical palpation. J Craniomaxillofac Surg 39(7): 534-537.

9. Som PM (1992) Detection of Metastasis in Cervical Lymph Nodes: CT and MR Criteria and Differential Diagnosis. 158(5): 961-969.

10. Haberal I, Celik H, Göçmen H, Akmansu H, Yörük M (2004) Which is important in the evaluation of metastatic lymph nodes in head and neck cancer: Palpation, ultrasonography, or computed tomography? Otolaryngol Head Neck Surg 130(2): 197-201.

11. Woolgar JA, Beirne JC, Vaughan ED, Lewis-Jones HG, Scott J, et al. (1995) Correlation of histopathologic findings with clinical and radiologic assessments of cervical lymph-node metastases in oral cancer. Int J Oral Maxillofac Surg 24(1): 30-37.

12. Close LG, Merkel M, Vuitch MF, Reisch J, Schaefer SD (1989) Computed Tomographic Evaluation of Regional Lymph Node Involvement In Cancer Of The Oral Cavity And Oropharynx. Head Neck 11(4): 309-317.

13. Al Kuliabi M (2007) A Method of Cervical Lymph Nodes Assessment in Oral Cancer Patients using Helical CT Scan. OMFS., U of K: Khartoum p. 65.

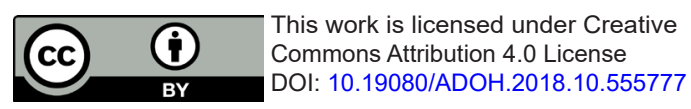

14. Paleri V, Urbano TG, Mehanna H, Repanos C, Lancaster J, et al. (1992) Management of cervical lymph nodes in patients with head and neck cancer. J Laryngol Otol 130(2): 161-169.

15. August M, Nguyen M (1994) Evaluation of metastatic neck disease by computed tomography. Int J Oral Maxillofac Surg 23(5): 290-293.

16. Bergman SA, Ord RA, Rothman M (1994) Accuracy of clinical examination versus computed tomography in detecting occult lymph node involvement in patients with oral epidermoid carcinoma. J Oral Maxillofac Sur 52(12): 1236-1239.

17. Shingaki S, Suzuki I, Nakajima T, Hayashi T, Nakayama H, et al. (1995) Computed tomographic evaluation of lymph node metastasis in head and neck carcinomas. J Craniomaxillofac Surg 23(4): 233-237.

18. Woolgar JA (1999) Pathology of the N0 neck. Br J Oral Maxillofac Surg 37(3): 205-209.

19. Freire AR, Lima EN, Almeida OP, Kowalski LP (2003) Computed tomography and lymphoscintigraphy to identify lymph node metastases and lymphatic drainage pathways in oral and oropharyngeal squamous cell carcinomas. Eur Arch Otorhinolaryngol 260 (3): 148-152.

20. Stern WB, Silver CE, Zeifer BA, Persky MS, Heller KS (1990) Computed tomography of the clinically negative neck. Head Neck 12(2): 109-113.

\section{Your next submission with Juniper Publishers will reach you the below assets}

- Quality Editorial service

- Swift Peer Review

- Reprints availability

- E-prints Service

- Manuscript Podcast for convenient understanding

- Global attainment for your research

- Manuscript accessibility in different formats

( Pdf, E-pub, Full Text, Audio)

- Unceasing customer service

Track the below URL for one-step submission https://juniperpublishers.com/online-submission.php 